\title{
Orthogonal beamforming using Gram-Schmidt orthogonalization for multi-user MIMO downlink system
}

\author{
Kunitaka Matsumura ${ }^{*}$ and Tomoaki Ohtsuki
}

\begin{abstract}
Simultaneous transmission to multiple users using orthogonal beamforming, known as space-division multipleaccess (SDMA), is capable of achieving very high throughput in multiple-input multiple-output (MIMO) broadcast channel. In this paper, we propose a new orthogonal beamforming algorithm to achieve high capacity performance in MIMO broadcast channel. In the proposed method, the base station generates a unitary beamforming vector set using Gram-Schmidt orthogonalization. We extend the algorithm of opportunistic SDMA with limited feedback (LF-OSDMA) to guarantee that the system never loses multiplexing gain for fair comparison with the proposed method by informing unallocated beams. We show that the proposed method can achieve a significantly higher sum capacity than LF-OSDMA and the extended LF-OSDMA without a large increase in the amount of feedback bits and latency.
\end{abstract}

Keywords: Multi-user MIMO, Gram-Schmidt orthogonalization, Space-division multiple-access (SDMA)

\section{Introduction}

In multiple-input multiple-output (MIMO) broadcast (downlink) systems, simultaneous transmission to multiple users, known as space-division multiple-access (SDMA), is capable of achieving very high capacity. In general, the capacity of SDMA can be considerably improved in comparison with time-division multipleaccess [1] because of multiuser diversity gain, which refers to the selection of users with good channels for transmission $[2,3]$. The optimal SDMA performance can be achieved by dirty paper coding (DPC) [4], however, implementation of DPC is infeasible since it requires complete channel state information (CSI) and high computational complexity. More practical SDMA algorithms are based on transmit beamforming, including zero forcing [5], minimum mean square error [6], and channel decomposition [7].

Various algorithms for limited feedback SDMA schemes have been proposed recently. When the number of users exceeds the number of antennas at the base station, a user scheduling algorithm should be jointly

\footnotetext{
*Correspondence: hassa83@z7.keio.jp

Department of Computer and Information Science, Keio University Hiyoshi 314-1, Kohoku-ku, Yokohama-shi, Kanagawa-ken 223-8522, Japan
}

designed with limited feedback multiuser precoding. For the opportunistic SDMA (OSDMA) algorithm proposed in [8], the feedback of each user is reduced to a few bits by constraining the choice of beamforming vector to a set of orthonormal vectors. In OSDMA, base station sends orthogonal beams, and each user reports the best beam and their signal-to-interference-plus-noise ratio (SINR) to the base station. The base station then schedules transmissions to some users based on the received SINR. For a large number of users, OSDMA ensures that the sum capacity increases with the number of users. However, the sum capacity of the OSDMA is limited if there are not a sufficient number of users.

To solve this problem, an extension of OSDMA, called OSDMA with beam selection (OSDMA-S), is proposed in [9]. OSDMA-S improves on OSDMA using beam selection to get capacity gain for any number of users in the system. However, multiple broadcast and feedback are required for implementing OSDMA-S, which incurs downlink overhead and feedback delay.

An alternative SDMA algorithm with orthogonal beamforming and limited feedback is proposed [10], called OSDMA with LF-OSDMA. LF-OSDMA results from the joint design of limited feedback, beam-forming

\section{SpringerOpen ${ }^{\circ}$}


and scheduling under the orthogonal beamforming constraint. In LF-OSDMA, each user selects the preferred beamforming vector with their normalized channel vector, called the Channel shape, using a codebook made up of multiple orthonormal vector sets. Then, each user sends back the index of the preferred beam vector as well as SINR to the base station. Using multi-user feedback and a criterion of maximum capacity, the base station schedules a set of simultaneous users with the beamforming vectors. More details of LF-OSDMA algorithm are stated in Section 3.

The simulation in [10] shows that LF-OSDMA can achieve significant gains in sum capacity with respect to OSDMA. However, LF-OSDMA does not guarantee the existence of $N_{t}$ (the number of transmit antennas) simultaneous users whose beam vectors belong to same orthonormal vector set, since each user selects a beamforming vector. This can result in the loss of multiplexing gain and hence the sum capacity of LF-OSDMA decreases for an increase of the number of subcodebooks.

In this paper, we propose a new orthogonal beamforming algorithm using Gram-Schmidt orthogonalization for achieving high capacity in MIMO broadcast channel. In this algorithm, the base station initially selects one or more users, and let them feed their full CSI back. Among the feedback users, the base station selects the one having highest channel gain. Using full CSI information, the base station generates beamforming vector for the selected user, and using Gram-Schmidt orthogonalization, the base station can generate a unitary orthogonal vector set. On the other hand, each user can generate the same unitary orthogonal vector set in the same way for the base station using CSI of the selected user from the base station. Each user selects the preferred beam from the generated beamforming vector set, and feeds the index of the preferred beam and quantized SINR back. Among feedback users, the base station schedules users using the criterion of maximizing sum capacity. More details of the proposed method are shown in Section 4. Because the base station generates the beamforming vector for the selected user and schedules the one, the proposed method is expected to achieve high sum capacity, though the number of feedback bits and the amount of latency increase in our system. For fair comparison of the amount of the latency, we extend the algorithm of LF-OSDMA to guarantee that the system never loses multiplexing gain in Section 5. Section 6 presents the analysis of the proposed method in terms of encoding, the effect of changing the number of initially selected users and the complexity at mobile terminal. In Section 7, we compare the number of feedback bits, the amount of latency, and the sum capacity of the proposed beamforming algorithm with LF-OSDMA and the extended LF-OSDMA. In the result, we show that the proposed method can achieve a significantly higher sum capacity than LF-OSDMA and the extended LF-OSDMA without a large increase in the amount of feedback bits and latency.

\section{System Model}

We consider a downlink multiuser multiple-antenna communication system, made up by a base station and $K$ active users. The base station is equipped with $N_{t}$ transmit antennas, and each user terminal is equipped with a single receive antenna. The base station can separate the multi-user data streams by beamforming, assigning a weight vector to each of $N_{t}$ active users. The weight vectors $\left\{\mathbf{w}_{n}\right\}_{n=1}^{N_{t}}$ are unitary orthogonal vectors, where $\mathbf{w}_{n} \in \mathcal{C}^{N_{t} \times 1}$ is a beamforming vector with $\left\|\mathbf{w}_{n}\right\|^{2}=1$. We assume that the equal power allocation over scheduled users. The received signal of the user $k$ is represented as

$$
y_{k}=\mathbf{h}_{k}^{T} \sum_{b \in B} \mathbf{w}_{b} x_{b}+n_{k}, \quad k \in B,
$$

where $\mathbf{h}_{k} \in \mathcal{C}^{N_{t} \times 1}$ is a channel gain vector of user $k$ with i.i.d. complex Gaussian entries $\sim \mathcal{C N}(0,1), x_{b}$ is the transmitted symbol with $\left|x_{b}\right|=1$ and $E\left[\left|x_{b}\right|\right]=1, B$ is the index set of scheduled users, and $n_{k}$ is complex Gaussian noise with zero mean and unit variance of user $k$. The superscript $T$ denotes the vector transpose. It is assumed that the user $k$ has perfect CSI $\mathbf{h}_{k}$.

\section{Conventional Orthogonal Beamforming}

An orthogonal beamforming and limited feedback algorithm were proposed in [10], called LF-OSDMA, which results from the joint design of limited feedback, beamforming and scheduling under the orthogonal beamforming constraint.

The CSI $\mathbf{h}_{k}$ can be decomposed into two components: gain and shape. Hence, $\mathbf{h}_{k}=g_{k} \mathbf{s}_{k}$ where $g_{k}=|| \mathbf{h}_{k} \|$ is the gain and $\mathbf{s}_{k}=\mathbf{h}_{k} /|| \mathbf{h}_{k}||$ is the shape. The channel shape is used for choosing weight vector, and the channel gain is used for computing SINR value. The user $k$ quantizes and sends back to the base station two quantities: the index of a selecting weight vector and the quantized SINR. We assume that a codebook is created using the method in IEEE 802.20 [11], which can be expressed as $\mathcal{F}=\left\{\mathbf{F}_{1}, \ldots \mathbf{F}_{M}\right\}$, where the subcodebook $\mathbf{F}_{i}$ is the unitary matrix and $M$ is the number of subcodebooks. By expressing each unitary matrix as $\mathbf{F}_{i}=\left\{\mathbf{f}_{i, 1}, \ldots, \mathbf{f}_{i, N_{t}}\right\}$, the preferred beam $\mathbf{q}_{k}$ selected by the user $k$, as a function of CSI's shape $\mathbf{s}_{k}$, is given by

$$
\mathbf{q}_{k}=\underset{\mathbf{f}_{i, j \in \mathcal{F}}}{\arg \max }\left|\mathbf{s}_{k}^{T} \mathbf{f}_{i, j}\right|
$$

where $\cdot{ }^{T}$ means transposition. To compute SINR, we define the quantization error as 


$$
\delta_{k}=\sin ^{2}\left(\angle\left(\mathbf{s}_{k}, \mathbf{q}_{k}\right)\right)
$$

It is clear that the quantization error is zero if $\mathbf{s}_{k}=\mathbf{q}_{k}$. The SINR for the user $k$ is a function of channel power $\rho_{k}=\left\|\mathbf{h}_{k}\right\|^{2}$ and the quantization error $\delta_{k}$

$$
\operatorname{SINR}_{k}=\frac{\rho_{k}\left(1-\delta_{k}\right)}{1 / \gamma+\rho_{k} \delta_{k}}
$$

where $\gamma$ is the input SNR. Each user feeds back its SINR along with the index of the preferred beam. Only the index of $\mathbf{q}_{k}$ needs to be sent back, because the quantization codebook $\mathcal{F}$ can be known a priori to both the base station and the users. We assume that the $\operatorname{SINR}_{k}$ is perfectly known to the base station by feedback processing. The same assumption is used in [8], [10]. Let the required number of bits for quantizing SINR be $Q_{\text {SINR, }}$, and the total amount of required feedback per user becomes $\log _{2}\left(N_{t} M\right)+Q_{\text {SINR }}$ bits.

Among feedback users, the base station schedules a subset of users using the criterion of maximizing sum capacity. Using the algorithm discussed in [10], [12], we group feedback users according to their quantized channel shapes as follows.

$$
\mathcal{L}_{i, j}=\left\{1 \leq k \leq K \mid \mathbf{q}_{k}=\mathbf{f}_{i, j}\right\}, 1 \leq i \leq M, 1 \leq j \leq N_{t}
$$

where $\mathbf{f}_{i, j} \in \mathcal{F}$ is the $i$ th beam vector in the $j$ th subcodebook. Among these subgroups, the one having the maximum sum capacity is scheduled, and base station selects the subcodebook having the maximum sum capacity for transmission. The resultant sum capacity can be written as

$$
\mathcal{C}=\max _{i=1, \ldots, M} \sum_{j=1}^{N_{t}} \log _{2}\left(1+\max _{k \in \mathcal{L}_{i, j}} \operatorname{SINR}_{k}\right) .
$$

If $\mathcal{L}_{i, j}$ is empty, we set $\max _{k \in \mathcal{L}_{i, j}} \operatorname{SINR}_{k}=0$.

In the situation that there is a large number of active users, LF-OSDMA can achieve high capacity. However, in the situation that there is a small number of active users, its capacity is limited because LF-OSDMA does not guarantee the existence of $N_{t}$ simultaneous users whose beam vectors belong to the same orthogonal vector set, in other words, there is an unallocated beam vector in the selected subcodebook. This can result in the loss of multiplexing gain and hence the sum capacity of LF-OSDMA decreases for an increase of the number of subcodebooks where there is a small number of active users.

\section{Proposed Orthogonal Beamforming Algorithm}

In this section, we propose a new orthogonal beamforming algorithm using Gram-Schmidt orthogonalization.
The proposed method is described from Steps I to VI as follows.

\section{Step I}

The base station initially selects $S$ users, and sends pilot signals to let all users estimate CSI, where $S$ is the number of users selected by the base station. In this paper, we assume that all users have perfect CSI. We denote the latency, until pilot signals are received by all users in the cell, by $\delta_{B C}$

\section{Step II}

Users who are initially selected by the base station feed back their full CSI, analog CSI. In this paper, we randomly selected the initial users who feed their full CSIs back, because at the initial step the base station does not have users' CSI and the proposed method does not want to increase the amount of feedback. We denote the latency, until selected users' feedback information are received by the base station, by $\delta_{\text {select }}$ and the number of feedback bits is $S Q_{\mathrm{CSI}}$ bits, where $Q_{\mathrm{CSI}}$ is the number of feedback bits of the full CSI.

\section{Step III}

Among the feedback users, the base station picks up the one having the highest channel gain from the initially selected users, which is defined as user $u$ that has CSI $\mathbf{h}_{u}$ and we refer to this user as the pivotal user. Using full CSI of user $u$, the base station generates a unitary orthogonal vector set, $\mathbf{W}=\left[\mathbf{w}_{1}, \mathbf{w}_{2}, \ldots, \mathbf{w}_{N_{t}}\right]$ as follows.

$$
\begin{aligned}
& \mathbf{w}_{1}=\mathbf{h}_{u} /\left\|\mathbf{h}_{u}\right\| \\
& \mathbf{X}=\mathbf{I}_{N_{t}}=\left[\mathbf{x}_{1} \mathbf{x}_{2} \ldots \mathbf{x}_{N_{t}}\right] \\
& \mathbf{w}_{l}=\frac{\mathbf{x}_{l}-\sum_{n=1}^{l-1} \mathbf{w}_{n}\left(\mathbf{w}_{n}^{H} \mathbf{x}_{l}\right)}{\left\|\mathbf{x}_{l}-\sum_{n=1}^{l-1} \mathbf{w}_{n}\left(\mathbf{w}_{n}^{H} \mathbf{x}_{l}\right)\right\|}, l=2, \ldots, N_{t}
\end{aligned}
$$

Where $\cdot{ }^{H}$ means Hermitian transposition. We assume $\mathbf{X}$ is $\left(N_{t} \times N_{t}\right)$ unit matrix, which is used for generating orthogonal weight vectors. Using Gram-Schmidt algorithm with $\mathbf{w}_{1}$, we generate orthogonal beams to $\mathbf{w}_{1}$. The vector $\mathbf{w}_{1}$ is the beamforming vector for user $u$, and the vector set of $\left[\mathbf{w}_{1}, \mathbf{w}_{2}, \ldots, \mathbf{w}_{N_{t}}\right]$ represents generated orthogonal beamforming vectors.

\section{Step IV}

The base station informs all users about information of $\mathrm{w}_{1}$. We denote the latency, until the information of $\mathbf{w}_{1}$ is received by all users in the cell, by $\delta_{\text {ad }}$, and the 
number of information bits is $Q_{\text {CSI }}$ bits which is the number of feedback bits of full CSI

\section{Step V}

Using information from the base station about $\mathbf{w}_{1}$, each user can generate the same unitary orthogonal vector set for the base station using (8) and (9). We assume that the algorithm for getting the unitary vector set is known a priori to both the base station and users. Then, each user selects the preferred beam $\mathbf{q}_{k}^{\prime}$ which is given by

$$
\mathbf{q}_{k}^{\prime}=\arg \max _{\mathbf{w}_{n} \in \mathbf{w}_{, \mathbf{w}_{n} \neq \mathbf{w}_{1}}}\left|\mathbf{s}_{k}^{T} \mathbf{w}_{n}\right| .
$$

The quantization error and SINR for the user $k$ is defined as

$$
\begin{aligned}
& \delta_{k}^{\prime}=\sin ^{2}\left(L\left(\mathbf{s}_{k}, \mathbf{q}_{k}^{\prime}\right)\right), \\
& \operatorname{SINR}_{k}^{\prime}=\frac{\rho_{k}\left(1-\delta_{k}^{\prime}\right)}{1 / \gamma+\rho_{k} \delta_{k}^{\prime}} .
\end{aligned}
$$

Each user feeds the quantized SINR' and the index of the preferred beam vector back. We denote the latency, until all users' feedback information are received by base station, by $\delta_{\text {all }}$. The number of feedback bits is $\log _{2} N_{t}+$ $Q_{\text {SINR }}$ bits.

\section{Step VI}

Among feedback users, the base station schedules users using the criterion of maximizing sum capacity. Certainly, the beam $\mathrm{w}_{1}$ is assigned by the user $u$, the pivotal user, because this beam is the beamforming vector for the user $u$.

\section{Extended Conventional Orthogonal Beamforming}

In this section, we extend the algorithm of conventional orthogonal beamforming to guarantee that there is no unallocated beam in the selected subcodebook. The proposed method always supports $N_{t}$ users, while the conventional LF-OSDMA cannot always support $N_{t}$ users, though its latency is smaller than that of the proposed method. Therefore, to compare the performance of those algorithms under more similar condition, we allow LF-OSDMA to support always $N_{t}$ users but with higher latency, which is the extended LF-OSDMA. The scheduling algorithm with the extended LF-OSDMA is described from Step 1 to Step 6 as follows.

\section{Step 1}

A base station sends pilot signals to let users estimate CSI. In this paper, we assume that all users have perfect
CSI $\mathbf{h}_{k}$. We denote the latency, until pilot signals are received by all users in the cell, by $\delta_{\mathrm{BC}}$

\section{Step 2}

Using CSI, each user chooses the preferred beam vector from codebook and calculates the receive SINR. Then, each user feeds back indexes of the preferred beam vector and quantized $\operatorname{SINR}_{k}$. We denote the latency, until all users' feedback information are received by base station, by $\delta_{\text {all }}$.

\section{Step 3}

Among feedback users, the base station schedules a subset of users, and selects the subcodebook having the maximum sum capacity.

So far, during Step 1 and Step 3, the algorithm is same as that of LF-OSDMA, and the extended part begins from Step 4 to Step 6 .

\section{Step 4}

If the selected subcodebook has an unallocated beam vector, the base station informs all users about indexes of the selected subcodebook and the unallocated beam vector. We denote the latency, until the information of the unallocated beam vector is received by all users in the cell, by $\delta_{\text {ad }}$, and the number of informed bits is $\log _{2}$ $M+N_{t}$ bits.

\section{Step 5}

Using information from the base station about the unallocated beam vector, each user can generate the unallocated beam vector set $\mathbf{F}_{m}=\left\{\mathbf{f}_{m, n}, \ldots\right\}, n \in\left\{1,2, \ldots, N_{t}\right\}$, and selects the preferred beam $\mathbf{q}_{k}^{\prime \prime}$ which can be given by

$$
\mathbf{q}_{k}^{\prime \prime}=\arg \max _{\mathbf{f}_{m, n} \in \mathbf{F}_{m}}\left|\mathbf{s}_{k}^{T} \mathbf{f}_{m, n}\right| .
$$

The quantization error and SINR for the user $k$ is defined as

$$
\begin{aligned}
& \delta_{k}^{\prime \prime}=\sin ^{2}\left(\angle\left(\mathbf{s}_{k}, \mathbf{q}_{k}^{\prime \prime}\right)\right), \\
& \operatorname{SINR}_{k}^{\prime \prime}=\frac{\rho_{k}\left(1-\delta_{k}^{\prime \prime}\right)}{1 / \gamma+\rho_{k} \delta_{k}^{\prime \prime}} .
\end{aligned}
$$

Each user feeds back the quantized SINR $R_{k}^{\prime \prime}$ and the index of the preferred beam vector. In this step, the latency is same as that of Step 2, and the number of feedback bits is $\log _{2} N_{t}+Q_{\text {SINR }}$ bits.

\section{Step 6}

Among feedback users, the base station assigns a user to the unallocated beam vector of the selected subcodebook using the criterion of maximizing sum capacity. 
The extended algorithm can guarantee the existence of $N_{t}$ simultaneous users, so even if there is a small number of users, and the extended LF-OSDMA can achieve high capacity. However, the extended LFOSDMA leads to the large increase in the number of feedback bits, and worsens system latency. We make comparisons of the number of the feedback bits and a system latency in Sect. 7.

\section{Analysis of the Proposed Method}

In this section, we analyze the proposed method in terms of encoding, the effect of changing the number of initially selected users $S$ and the complexity at mobile terminal.

\subsection{Encoding of the proposed method}

In this subsection, we evaluate the capacity performance of the proposed method when CSI is quantized by a random vector quantization codebook, because the feedback of the full CSI results in a large amount. The size of the codebook is $2^{Q C S I}$ where $Q_{C S I}$ is the number of feedback bits of the CSI. Figure 1 shows the sum capacity of the proposed method for different codebook sizes, $Q_{\text {CSI }}=\{5,10,15,20$, analog CSI $\}$, for an increase of users. The number of transmit antennas is $N_{t}=4$, SNR is $5 \mathrm{~dB}$ and the number of the initially selected user is $S=1$. We come up with the results based on Monte Carlo simulation.

As the codebook size becomes larger, the sum capacity of the proposed method increases. This is because the quantization error of CSI becomes smaller, as the codebook size becomes larger. As observed from Figure 1, 15 bits of the CSI feedback causes only marginal loss in sum capacity with respect to the analog CSI feedback. Such loss is negligible for 20-bits feedback. Therefore, the feedback by the codebook of $Q_{\mathrm{CSI}}=20$ from the initially selected users is as good as the analog CSI case. Thus, in this paper, we assume that the number of the feedback bits of the full CSI is $Q_{\text {CSI }}=20$ when we evaluate the feedback bits. Actually, the codebook of $Q=20$ is not preferable in practice because of the large complexity at the mobile terminal side.

\subsection{Effect of changing the number of initially selected users}

In this section, we show the capacity result and the number of feedback bits of the proposed method with the increase of the number of initially selected users by the base station. Note that $S$ affects the amount of feedback, but is not dependent on the number of transmit antennas $N_{t}$.

Figure 2 shows the sum capacity of the proposed method for different number of initially selected users, $S=1,3,5$, all active users, for an increase of users. The number of transmit antennas is $N_{t}=4$ and the SNR is 5 $\mathrm{dB}$. We came up with the results of the capacity based on Monte Carlo simulation. By Monte Carlo simulation, we generate each user's flat Rayleigh fading channel and AWGN. Based on these values, we calculate each user's SINR using (4), (12) or (15). Using the SINR and (6), we calculate sum capacity. For the increase of the number of initially selected users, the sum capacity of the proposed method increases, however, the rate of improvement of the sum capacity decreases. The difference of the sum capacity between $S=1$ and $S=2$ is about 0.4 bits $/ \mathrm{Hz}$ at $K=100$, but there is little difference between $S=2$ and $S=3$. Therefore, $S=1$ or $S=2$ are practical.

Figure 3 shows the number of feedback bits of the proposed method with the increase of the number of initially selected users by the base station. We calculate

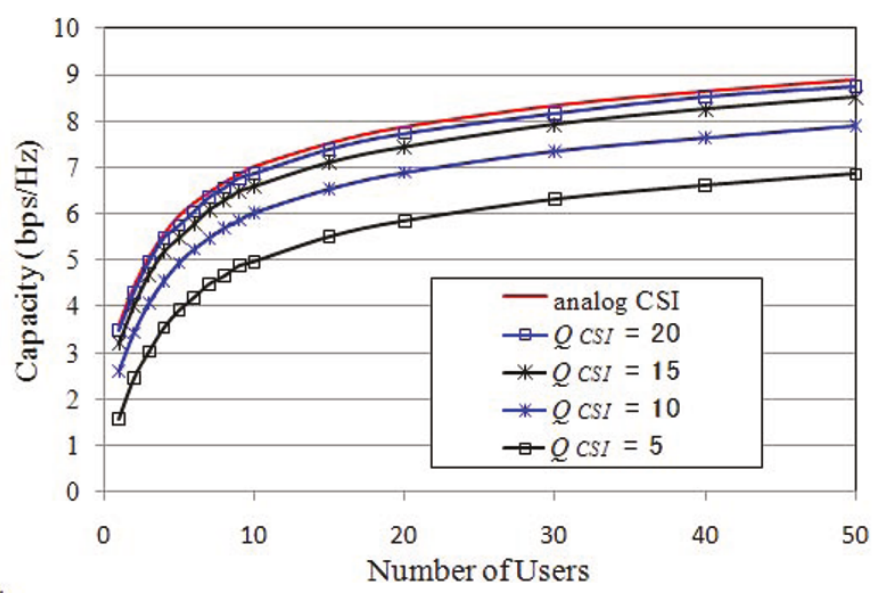

Figure 1 Sum capacity of the proposed method for different number of codebook size, $Q_{C S I}=\{5,10,15,20$, analog CSI $\}$, for an increase of users, the number of transmit antenna is $N_{t}=4$, SNR is $5 \mathrm{~dB}$ and the initially selected user is $S=1$. 


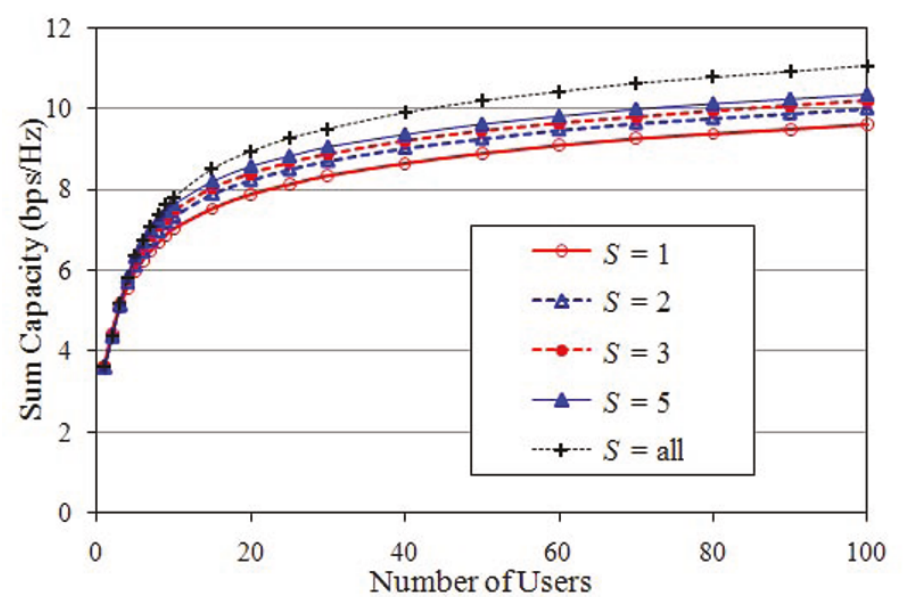

Figure 2 Sum capacity of the proposed method for different number of initially selected users $S$, SNR $=\mathbf{5} \mathrm{dB}$, and the number of transmit antennas are $N_{t}=4$.

the number of feedback bits based on the analytic formula, and there are two times for the base station to receive feedback from active users. First time, the initially selected $S$ users feed their full CSIs back to the base station, and the number of the first-feedback bits is $S Q_{\text {CSI }}$ bits, where $Q_{\text {CSI }}$ is the number of feedback bits of the full CSI per user. Thus, the number of the initially selected users, $S$, affects the number of first-feedback bits, but is not dependent on the number of the active users, $K$ nor the number of transmit antennas $N_{t}$. Second time, the base station receives feedback about the selected beamforming vector from all active users other than the pivotal user, and the number of the secondfeedback bits is $(K-1)\left(\log _{2} N_{t}+Q_{\text {SINR }}\right)$, where $Q_{\text {SINR }}$ is the number of feedback bits of quantizing SINR. Thus, the number of active users, $K$, affects the number of second-feedback bits, but is not dependent on the number of the initially selected users, $S$.

When $S=$ all active users, the proposed method produces explosive growth of the number of feedback bits, because all users in the cell feed back their full CSI. When $S \neq$ all active users, the difference of the number of feedback bits is constant, which represents that of the full CSI from initially selected users. If we increase the number of initially selected users $S$ by 1 , the number of feedback bits is increased by $Q_{\mathrm{CSI}}=20$ bits.

\subsection{Complexity at the mobile terminal}

In this section, we show the complexity of the proposed method at the mobile terminal side in comparison with LF-OSDMA. We evaluate the complexity by the number of scalar multiplications and square roots. Table 1

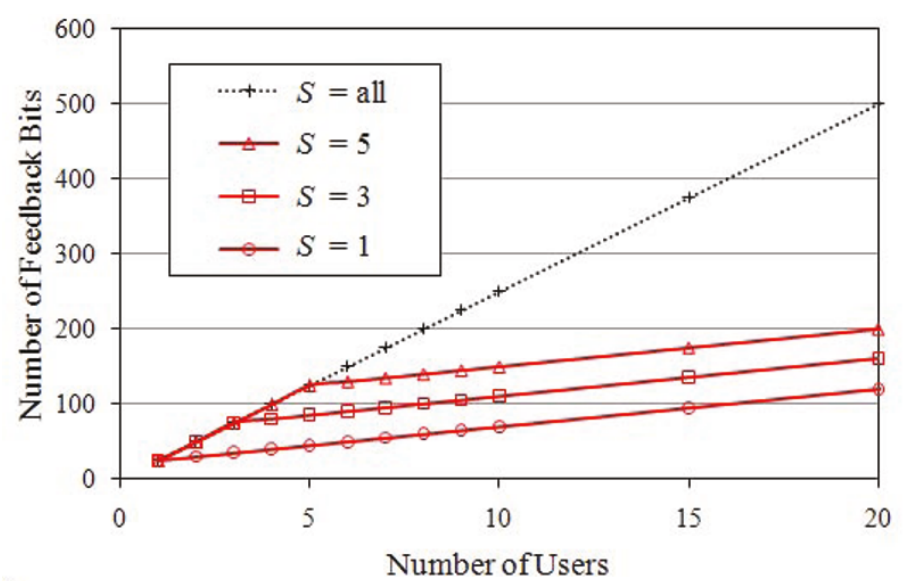

Figure 3 Number of feedback bits of the proposed method, LF-OSDMA, and the extended LF-OSDMA for an increase of the number of users $K, Q_{\mathrm{CSI}}=20, S$ is the number of users selected by the base station, $M$ is the number of subcodebooks, $Q_{\mathrm{SINR}}=3$ and the number of transmit antennas is $N_{t}=4$. 
Table 1 The complexity of LF-OSDMA at users

\begin{tabular}{llccc}
\hline & & \multicolumn{3}{c}{ The number of operators } \\
\cline { 3 - 5 } & & & $(\boldsymbol{M}=\mathbf{8})$ & $(\boldsymbol{M}=\mathbf{1})$ \\
\hline Selection of the BF vector & Multiplications & $N_{t} M\left(4 N_{t}+2\right)$ & 576 & 72 \\
\cline { 2 - 5 } & Square roots & $N_{t} M$ & 32 & 4 \\
\hline
\end{tabular}

shows the complexity of LF-OSDMA at users. In LFOSDMA, each user has $N_{t} M\left(4 N_{t}+2\right)$ multiplications and $N_{t} M$ square roots when selects the beamforming vector from the codebook, where $N_{t}$ is the number of transmit antennas and $M$ is the number of subcodebooks. Table 2 shows the complexity of the proposed method. In the proposed method, the implementation of each user consists of two stages: generation of the same unitary orthogonal vector set for the base station using (8) and (9), and the selection of the beamforming vector. We neglect the complexity of the initially selected users, because they feed the analog CSI back. In the former, (8) has $\left(N_{t}-1\right) ! 8 N_{t}+\left(N_{t}+1\right)$ multiplications and one square root. In the latter, each user has $\left(N_{t}-1\right)\left(4 N_{t}+2\right)$ multiplications and $\left(N_{t}-1\right)$ square roots.

\section{Performance Comparison}

\subsection{Feedback comparison}

In this subsection, we compare the number of feedback bits among the proposed method, LF-OSDMA and the extended LF-OSDMA. We calculate the number of the feedback bits based on the analytic formula, and summarize them in Table 3. Actually, the feedback bits of the extended LF-OSDMA in Step 5 cannot be calculated by the analytic formula, and we assume it $K\left(\log _{2} N_{t}+\right.$ $\left.Q_{\text {SINR }}\right)$ this time. Figure 4 shows the number of feedback bits for an increase of the number of users until $K=20$. To compare the extended LF-OSDMA with the proposed method in terms of latency, we assume the extended LF-OSDMA always informs all users about the index of the unallocated beam vector. Thus, every system in this paper has the linearly-increasing number of feedback bits. We assume that the number of transmit antennas is $N_{t}=4$, the number of feedback bits of the full channel information is $Q_{\text {CSI }}=20$ bits and that of quantizing SINR is $Q_{\text {SINR }}=3$ bits [10].
Figure 4 shows that the proposed method needs fewer number of feedback bits than the extended LF-OSDMA, and needs almost the same number of feedback bits as LF-OSDMA. We can also observe from Figure 4 that the difference of the number of feedback bits between the proposed method and LF-OSDMA for $M=1$ is constant, which represents the number of feedback bits of the full CSI from initially selected users. If there is a large number of users, e.g. $K=100$, the proposed method needs much fewer number of feedback bits than the extended LF-OSDMA and LF-OSDMA with $M=8$. Therefore, the increase of the number of the feedback bits for the proposed method against that of LFOSDMA with $M=1$ is not large compared with that of LF-OSDMA with $M=8$ and extended LF-OSDMA.

\subsection{Latency comparison}

In this section, we compare the latency among the proposed method, LF-OSDMA, and the extended LFOSDMA. Table 4 lists the comparison of system latency. $\delta_{\mathrm{BC}}$ is the latency that is the amount of time from the sending pilot signals of the base station to the receiving of all users in the cell; $\delta_{\text {all }}$ is the latency that is the amount of time from the sending feedback information of all users to the receiving of the base station; $\delta_{\text {ad }}$ is the latency that is the amount of time from the sending the information of unallocated beam vector of the base station to the receiving of all users in the cell; and $\delta_{\text {select }}$ is the latency that is the amount of time from the sending the feedback information of the initially selected users to receiving of the base station.

Table 4 shows that the extended LF-OSDMA and the proposed method have to tolerate higher latency than that of LF-OSDMA. In practical systems, $\delta_{B C}$ and $\delta_{\text {ad }}$ are much lower than $\delta_{\text {all }}$ or $\delta_{\text {selec }}$, because $\delta_{\mathrm{BC}}$ and $\delta_{\text {ad }}$ use a downlink broadcast channel. In addition, if there

Table 2 The Complexity of the Proposed Method at Users

\begin{tabular}{ccc}
\hline \multirow{2}{*}{ Generation of the vectors } & Multiplications & $\left(N_{t}-1\right) ! 8 N_{t}+\left(N_{t}+1\right)$ \\
\cline { 2 - 3 } & Square roots & 1 \\
\hline Selection of the BF vector & Multiplications & $\left(N_{t}-1\right)\left(4 N_{t}+2\right)$ \\
\cline { 2 - 3 } & Square roots & $\left(N_{t}-1\right)$ \\
\hline Total & Multiplications & \\
\hline
\end{tabular}


Table 3 Comparison of the Feedback Bits

\begin{tabular}{cccc}
\hline & LF-OSDMA & Extended LF-OSDMA & Proposed method \\
\hline Step2 or Step II & $K\left(\log _{2} N_{t} M+Q_{\text {SINR }}\right)$ & $K\left(\log _{2} N_{t} M+Q_{S I N R}\right)$ & $S Q_{C S I}$ \\
\hline Step 5 or Step V & & $K\left(\log _{2} N_{t}+Q_{\text {SINR }}\right)$ & $(K 1)\left(\log _{2} N_{t}+Q_{S I N R}\right)$ \\
\hline
\end{tabular}

is a large number of users in the cell, $\delta_{\text {selec }}$ is much smaller than $\delta_{\text {all }}$. Therefore, the increase of the latency for the proposed method against LF-OSDMA is not large. However, the increase of the latency affects the capacity of the proposed method, particularly in case of high mobility.

\subsection{Capacity comparison}

In this section, we show the capacity result of the proposed beamforming algorithm. Figure 5 compares the sum capacity of the proposed method with that of LFOSDMA and the extended LF-OSDMA for an increase of the number of users. The number of transmit antennas is $N_{t}=4$ and SNR is $5 \mathrm{~dB}$. Moreover, the number of subcodebooks is $M=\{1,8\}$ for LF-OSDMA and the extended LF-OSDMA. The number of initially selected users by the base station is $S=\{1,2\}$ for the proposed method. We came up with the results of the capacity based on Monte Carlo simulation. By Monte Carlo simulation, we generate each user's flat Rayleigh fading channel and AWGN. Based on these values, we calculate each user's SINR using (4), (12) or (15). Using the SINR and (6), we calculate sum capacity.

Firstly, the proposed method achieves a significantly higher sum capacity than LF-OSDMA and the extended LF-OSDMA for any number of users. This is because in the proposed method, the base station generates the beamforming vector for the initially selected user using full CSI, and allocates other users to the vectors that do not cause interference to the beamforming vector for the initially selected user. The sum capacity of LF-OSDMA decreases for an increase of the number of subcodebooks where there is a small number of active users. On the other hand, the extended LF-OSDMA improves the sum capacity on that of LF-OSDMA for the small number of users, because the extended LF-OSDMA guarantees that there is no unallocated beam in the selected subcodebook. However, for a large number of users, there is little difference in the sum capacity between LF-OSDMA and the extended LF-OSDMA, because LF-OSDMA can sufficiently get the multiplexing gain since there is a large number of users. At $K=20$, the capacity gain of the proposed method with respect to LF-OSDMA with $M=1$ is $2 \mathrm{bps} / \mathrm{Hz}$ and with respect to the extended LF-OSDMA with $M=8$ is 1 bits $/ \mathrm{Hz}$. At $K=100$, the proposed method also improves the sum capacity of LF-OSDMA and the extended LF-OSDMA by $0.5 \mathrm{bps} / \mathrm{Hz}$. In the result, the proposed method can achieve a significantly higher sum capacity than LF-OSDMA and the extended LF-OSDMA without a large increase in the amount of feedback bits and latency.

\subsection{Cumulative distribution function}

In this section, we show the cumulative distribution function (CDF) of the capacity on a per-user basis, because it is important for a system designer to consider this performance. We come up with the results based on the Monte Carlo simulation. Figure 6 compares the CDF of the proposed method with that of LF-OSDMA and the extended LF-OSDMA. The number of transmit

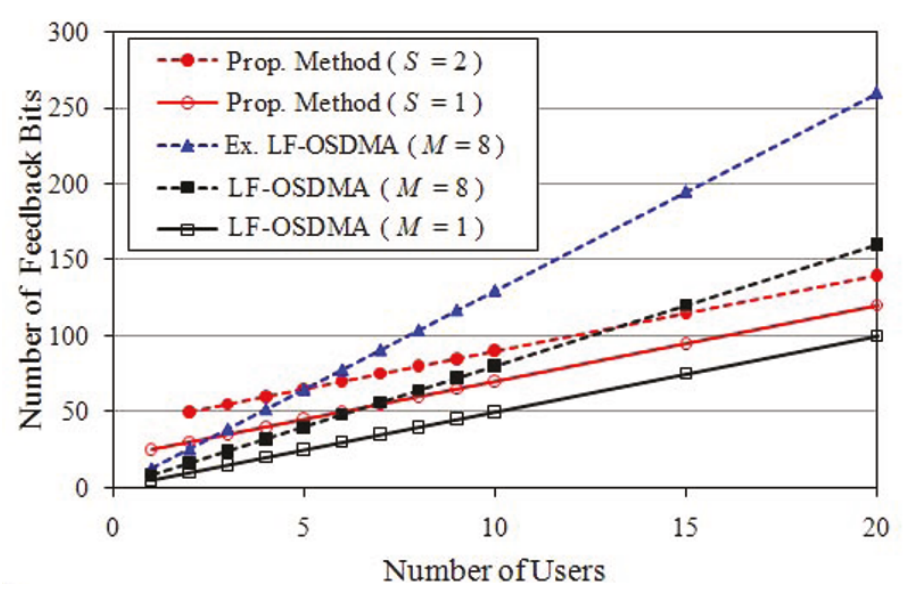

Figure 4 Number of feedback bits of the proposed method for different number of initially selected users $S, Q_{\mathrm{CSI}}=20, Q_{\mathrm{SINR}}=3$ and the number of transmit antennas is $N_{t}=4$. 
Table 4 Comparison system latency

\begin{tabular}{cccc}
\hline & LF-OSDMA & $\begin{array}{c}\text { Extended } \\
\text { LF-OSDMA }\end{array}$ & $\begin{array}{c}\text { Proposed } \\
\text { method }\end{array}$ \\
\hline BS $\rightarrow$ User & $\delta_{\mathrm{BC}}$ & $\delta_{\mathrm{BC}}$ & $\delta_{\mathrm{BC}}$ \\
$($ Step 1 or Step I) & $\delta_{\text {all }}$ & $\delta_{\text {all }}$ & $\delta_{\text {ad }}$ \\
\hline $\begin{array}{c}\text { User } \rightarrow \text { BS } \\
(\text { Step } \text { or Step II) }\end{array}$ & $\delta_{\text {ad }}$ & $\delta_{\text {all }}$ \\
\hline BS $\rightarrow$ User & $\delta_{\text {all }}$ & \\
\hline Step 4 or Step IV) & & \\
\hline User $\rightarrow$ BS & & \\
\hline
\end{tabular}

antennas is $N_{t}=4, \mathrm{SNR}$ is $5 \mathrm{~dB}$ and the number of users is $K=50$. In this simulation, we also randomly selected the initially selected users who feed their full CSIs back, and $S$ affects the amount of feedback, but is not dependent on the number of transmit antenna $N_{t}$.

Figure 6 shows that the proposed method has a higher variance of the capacity on a per-user basis than LFOSDMA and the extended LF-OSDMA. All users of LFOSDMA and the extended LF-OSDMA achieve the capacity between 1 and $3 \mathrm{bps} / \mathrm{Hz} / \mathrm{User}$. On the other hand, in the proposed method, the users achieve the capacity higher than or equal to those in LF-OSDMA. In addition, the variance of the capacity in the proposed method is larger as well. These are because in the proposed method, the pivotal user can have a much higher capacity than the users in LF-OSDMA and the extended LF-OSDMA. In addition, for the selected users other than the pivotal user, the amount of mismatch between each user's channel and the selected beamforming vector is about the same as that in the conventional algorithms. Therefore, the proposed method achieves the improvement of the capacity for the whole of the system compared with LF-OSDMA and the extended LF-
OSDMA without the loss of the capacity on a per-user basis, though the variance of the capacity on a per-user basis becomes large.

\section{Conclusion}

In this paper, we proposed a new orthogonal beamforming algorithm for the MIMO BC aiming to achieve high capacity performance for any number of users. In this algorithm, we do not use codebook, and the base station generates a unitary beamforming vector set using GramSchmidt orthgonalization using the beamforming vector for the pivotal user. Then, the pivotal user can use the optimal beamforming vector because of using analog value of the actual CSI. The proposed method increases the number of feedback bits and the amount of latency. For fair comparison about the amount of latency, we extend the algorithm of LF-OSDMA to guarantee that the system never loses multiplexing gain. Finally, we compare the number of feedback bits, the amount of latency, and the sum capacity of the proposed beamforming algorithm with LF-OSDMA and the extended LF-OSDMA. We showed that the proposed method can achieve a significantly higher sum capacity than LF-

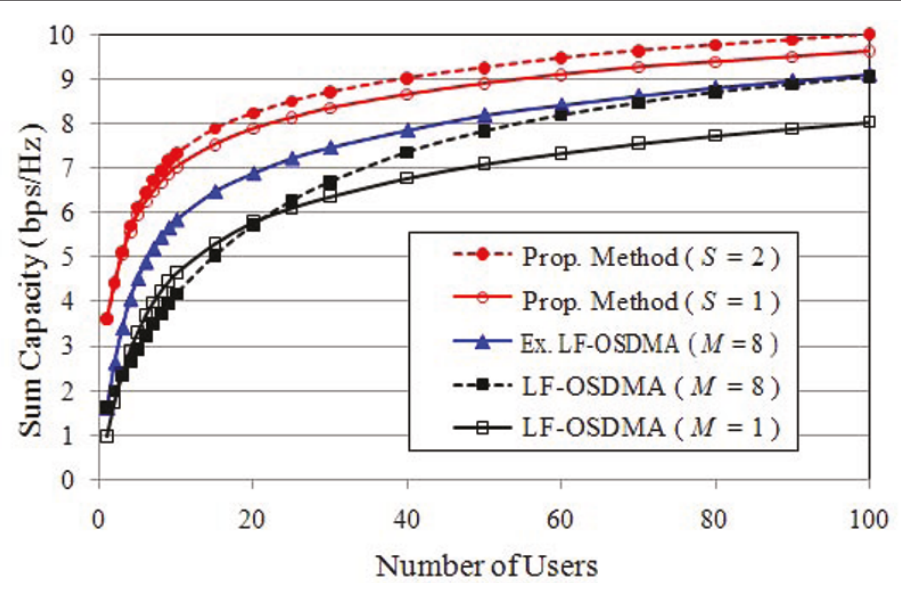

Figure 5 Sum capacity comparison among the proposed method, LF-OSDMA, and the extended LF-OSDMA for an increase of the number of users $K$; SNR $=5 \mathrm{~dB} ; S$ is the number of users selected by the base station, $M$ is the number of subcodebooks, and the number of transmit antennas is $N_{t}=4$. 


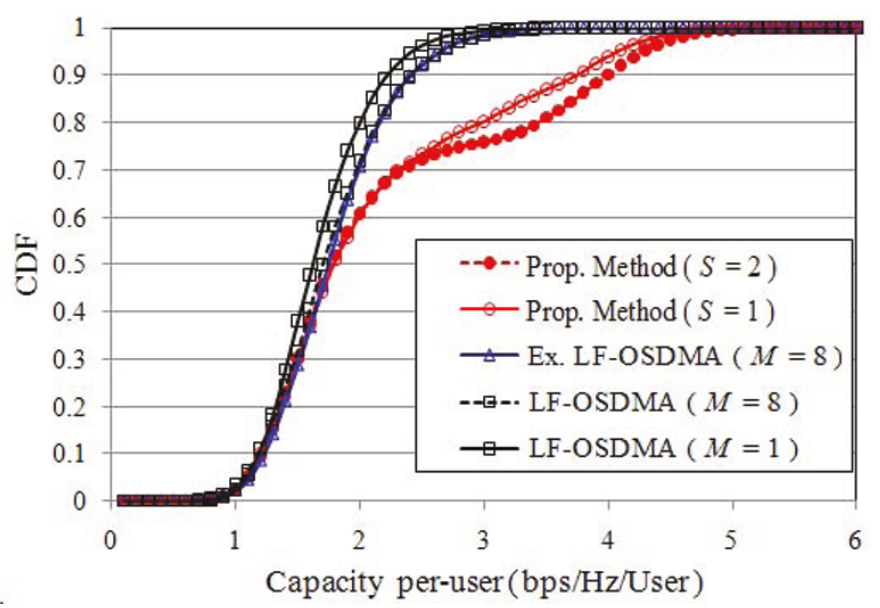

Figure 6 CDF of the capacity on a per-user basis for the proposed method, LF-OSDMA, and the extended LF-OSDMA, $N_{t}=4$, SNR is 5 $\mathrm{dB}$ and the number of users is $K=50$.

OSDMA and the extended LF-OSDMA without a large increase in the amount of feedback bits and latency. In this paper, we adopt IEEE 802.20 codebook for the LFOSDMA, but there may exist optimal codebook for LFOSDMA. In addition, the high correlation among the users's channels may affect the capacity of the proposed method largely. We want to examine these point in our future research.

\section{Abbreviations}

CDF: cumulative distribution function; CSI: channel state information; DPC: dirty paper coding; LF-OSDMA: SDMA with limited feedback; MIMO: multiple-input multiple-output; OSDMA: opportunistic SDMA; SDMA: spacedivision multiple-access; SINR: signal-to-interference-plus-noise ratio.

\section{Competing interests}

The authors declare that they have no competing interests.

Received: 1 November 2010 Accepted: 18 July 2011

Published: 18 July 2011

\section{References}

1. P Viswanath, D Tse, Sum capacity of the vector Gaussian broadcast channel and uplink-downlink duality. IEEE Trans Inf Theory. 49(8), 1912-21 (2003) doi:10.1109/TIT.2003.814483

2. TYoo, A Goldsmith, On the optimality of multi-antenna broadcast scheduling using zero-forcing beamforming. IEEE J Sel Areas Commun. 24(3), 528-541 (2006)

3. Z Shen, R Chen, JG Andrews, RW Heath Jr, BL Evans, Low complexity user selection algorithms for multiuser MIMO systems with block diagonalization. IEEE Trans Signal Process. 54(9), 3658-3663 (2006)

4. M Costa, Writing on dirty paper. IEEE Trans Inf Theory. 29(3), 439-441 (1983). doi:10.1109/TIT.1983.1056659

5. G Dimic, ND Sidiropoulos, On downlink beamforming with greedy user selection: performance analysis and a simple new algorithm. IEEE Trans Signal Process. 53(10), 3857-3868 (2005)

6. S Serbetlli, A Yener, Transceiver optimization for multiuser MIMO systems. IEEE Trans Signal Process. 52(1), 214-226 (2004). doi:10.1109/ TSP.2003.819988

7. YS Choi, S Alamouti, $V$ Tarokh, Complementary beamforming: new approaches. IEEE Trans Commun. 54(1), 41-50 (2006)
8. M Sharif, B Hassibi, On the capacity of MIMO broadcast channels with partial side information. IEEE Trans Inf Theory. 51(2), 506-522 (2005). doi:10.1109/TIT.2004.840897

9. W Choi, A Frenza, JG Andrews, RW Heath Jr, Opportunistic space division multiple access with beam selection. IEEE Trans Commun. 55(12), 2371-2380 (2007)

10. K-B Huang, RW Heath Jr, JG Andrews, Performance of orthogonal beamforming for SDMA with limited feedback. IEEE Trans Veh Technol. 58(1), 152-164 (2009)

11. IEEE 802.20 C802.20-06-04, Part 12: Precoding and SDMA codebooks (2006)

12. K Huang, RW Heath Jr, JG Andrews, Space division multiple access with a sum feedback rate constraint. IEEE Trans Signal Process. 55(7), 3879-3891 (2007)

doi:10.1186/1687-1499-2011-41

Cite this article as: Matsumura and Ohtsuki: Orthogonal beamforming using Gram-Schmidt orthogonalization for multi-user MIMO downlink system. EURASIP Journal on Wireless Communications and Networking 2011 2011:41

\section{Submit your manuscript to a SpringerOpen ${ }^{\circ}$ journal and benefit from:}

- Convenient online submission

- Rigorous peer review

- Immediate publication on acceptance

- Open access: articles freely available online

- High visibility within the field

- Retaining the copyright to your article

Submit your next manuscript at $\boldsymbol{s p r i n g e r o p e n . c o m ~}$ 